\title{
Oncogene K-Ras
}

National Cancer Institute

\section{Source}

National Cancer Institute. Oncogene K-Ras. NCI Thesaurus. Code C16766.

Human Oncogene K-Ras is a mutated variant of KRAS2 Gene, which encodes two alternative isoforms of monomeric p21 K-RAS protein, a monomeric GTPase involved in transmembrane signal transduction that alternates between inactive GDP-bound and active GT P-bound forms. K-Ras is activated by a guanine nucleotide-exchange factor and inactivated by a GT Pase-activating protein. Mitogen-stimulated RAS stabilizes MYC protein and enhances MYC accumulation by the RAS/RAF/MAPK pathway, which appears to inhibit the proteasome-dependent degradation of MYC. Implicated in a variety of human tumors, mutations of specific amino acids activate RAS to transform cells. KRAS is involved in malignancy much more often than is HRAS. Oncogene K-Ras disrupts normal cell function. 